\title{
Increased blood viscosity is associated with reduced renal function and elevated urinary albumin excretion in essential hypertensives without chronic kidney disease
}

\author{
Hiroko Sugimori, Fumihiro Tomoda, Tsutomu Koike, Hiroko Kurosaki, Toshitaka Masutani, Maiko Ohara, \\ Satoshi Kagitani and Hiroshi Inoue
}

Increased blood viscosity reduces blood flow and elevates vascular resistance in the cardiovascular system. The aim of this study was to elucidate how blood viscosity could affect renal function and eventually contribute to renal damage in essential hypertensives (EHT). In 164 untreated EHT without apparent renal damage (96 men, $56 \pm 12$ years old, creatinine clearance $123 \pm 33 \mathrm{ml} \mathrm{min}^{-1}$ per $1.73 \mathrm{~m}^{2}$ and urinary albumin excretion $19 \pm 19 \mathrm{mg}$ per day), blood and plasma viscosity was determined using a falling ball microviscometer. Blood viscosity correlated negatively with creatinine clearance $(r=-0.185, P=0.018)$ and positively with urinary albumin excretion $(r=0.253, P=0.001)$. This indicated that increased blood viscosity is associated with reduced renal function and worsening of albuminuria in EHT. Stepwise multiple regression analysis identified blood viscosity as an independent determinant of creatinine clearance $\left(R^{2}=0.058\right)$ and urinary albumin excretion $\left(R^{2}=0.216\right)$. In conclusion, increased blood viscosity may be a risk for development of renal disease in EHT.

Hypertension Research (2013) 36, 247-251; doi:10.1038/hr.2012.172; published online 15 November 2012

Keywords: albuminuria; blood viscosity; essential hypertension; renal function

\section{INTRODUCTION}

According to the Hagen-Poiseuille law, ${ }^{1}$ an increase in blood viscosity reduces blood flow and elevates vascular resistance in the cardiovascular system. This thesis is compatible with the clinical observation that conditions associated with increased blood viscosity, for example, hyperviscosity syndrome, often exhibit elevated total vascular resistance and decreased intravascular blood flow, leading to systemic hypertension and ischemic organ damage, respectively. ${ }^{2,3}$

In the renal microcirculation, hematocrit begins to rise in the afferent arterioles and reaches a maximal level in the efferent arterioles during the course of fluid loss into Bowman's space by glomerular filtration. ${ }^{4,5}$ Because hematocrit is the most important determinant of blood viscosity, ${ }^{1,2}$ blood is likely to be the most viscous in the vascular beds of the kidney in comparison with other organs. Therefore, in the clinical settings with elevated blood viscosity, harmful effects of blood viscosity are expected to manifest particularly in the kidney. In fact, hemorheological abnormalities may have a role in the pathogenesis of proteinuria (a marker of glomerular damage) in such patients with increased blood viscosity as cyanotic congenital heart disease, diabetes mellitus or central obesity. ${ }^{6-8}$ Additionally, increased viscosity of contrast media is one of the factors contributing to the pathogenesis of contrast-induced nephropathy. ${ }^{9,10}$
It is well known that essential hypertensives (EHT) have increased blood viscosity, ${ }^{11-14}$ which is associated with poor prognosis in EHT, because hyperviscosity is often accompanied by severe hypertension and cardiovascular complications including left ventricular hypertrophy. ${ }^{13,14}$ However, it has not been elucidated yet how blood viscosity affects renal hemodynamics and contributes to the genesis of renal damage in EHT. In the present study, therefore, associations of blood viscosity with renal function and urinary albumin excretion were investigated in untreated EHT without apparent kidney disease.

\section{METHODS}

The subjects comprised 164 consecutive and untreated EHT. The study design was approved by the Ethics Committee at University of Toyama and the patients were enrolled into the study after giving informed consent. The diagnosis of hypertension was made on the basis of a sitting diastolic blood pressure measured with a sphygmomanometer more than $90 \mathrm{~mm} \mathrm{Hg}$ and/or systolic blood pressure more than $140 \mathrm{~mm} \mathrm{Hg}$ on three separate occasions over a 4-week period. No patient had secondary hypertension, diabetes mellitus or other clinically evident cardiovascular diseases. Chronic kidney disease, defined by overt proteinuria (that is, urinary albumin excretion $>300 \mathrm{mg}$ per day), decreased renal function (that is, creatinine clearance $<60 \mathrm{mlmin}^{-1}$ per $1.73 \mathrm{~m}^{2}$ ) or both, ${ }^{15}$ was not observed in any patients. 
Patients were instructed not to take food, alcohol, caffeine or smoke within $12 \mathrm{~h}$ before blood samplings, although water ingestion was not restricted. In the morning, venous blood was collected in tubes containing potassium EDTA for determination of blood and plasma viscosity after a 30-min supine rest at the outpatient clinic. Blood samples were also taken for determinations of (1) factors associated with blood viscosity or plasma viscosity, including hematological variables, plasma fibrinogen, serum levels of total cholesterol, triglyceride and high-density lipoprotein (HDL) cholesterol, fasting levels of blood glucose and plasma insulin, and serum high-sensitive C-reactive protein; ${ }^{16-19}$ (2) renal function and (3) plasma nitrates and nitrites $\left(\mathrm{NO}_{\mathrm{x}}\right)$, a marker for endothelial nitric oxide (NO) production. Subsequently, $24 \mathrm{~h}$ urine was collected for measurements of creatinine clearance and urinary albumin excretion. Creatinine clearance was calculated as follows: creatinine clearance $\left(\mathrm{ml} \mathrm{min}{ }^{-1}\right.$ per $\left.1.73 \mathrm{~m}^{2}\right)=$ [urinary creatinine $\left(\mathrm{mg} \mathrm{dl}^{-1}\right) \times$ urinary volume $(\mathrm{ml}$ per day $)] /\left[\right.$ serum creatinine $\left.\left(\mathrm{mg} \mathrm{dl}^{-1}\right) \times 1440(\min )\right] /[$ body surface area $\left(\mathrm{m}^{2}\right) / 1.73$ ]. In all patients, incomplete urinary sampling was denied because of urinary creatinine excretion $>3 \mathrm{mmol}$ per day. ${ }^{20}$

For hemorheological measurements, whole blood viscosity and plasma viscosity were determined using a falling ball microviscometer (AMVn-200, Anton Paar, Austria). ${ }^{21}$ This instrument comprises a glass capillary tube of $0.16 \mathrm{~mm}$ internal diameter, containing a metallic ball of $0.15 \mathrm{~mm}$ diameter. Fluid viscosity is determined by the falling time of the ball in the tube inclined at 70 degrees, filled with blood or plasma at $20^{\circ} \mathrm{C}^{22,23}$ In this setting, fluid viscosity is measured at a high shear rate between 2500 and $3000 \mathrm{~s}^{-1}$, which allows measurement of blood viscosity in a completely disaggregated structure. $^{23}$ In nine healthy volunteers (five men and four women), reproducibility of blood viscosity and plasma viscosity was determined on four occasions at 1-week intervals. The within-subject coefficient of variance for these measurements was less than $5 \%$.

Biochemical parameters were measured using conventional laboratory techniques. Plasma $\mathrm{NO}_{\mathrm{x}}$ was determined by colorimetric assay kit using Griess reagent (Cayman Chemical Company, Ann Arbor, MI, USA). Insulin resistance was estimated according to the homeostasis model assessment (HOMA) as follows: HOMA index of insulin resistance $($ HOMA-IR) $=$ fasting blood glucose $\left(\mathrm{mg} \mathrm{dl}^{-1}\right) \times$ fasting plasma insulin $\left(\mu \mathrm{IU} \mathrm{dl}^{-1}\right) / 405$. Urinary albumin concentration was determined using immunoturbidimetry.

In addition, to examine the difference in hemorheological variables between EHT and normotensive subjects, blood and plasma viscosity was measured in 23 normotensive healthy subjects.

\section{Data analyses}

Data are presented as mean \pm s.d. The association of blood viscosity with creatinine clearance or urinary albumin excretion was determined by Pearson's correlation. In addition, to investigate whether blood viscosity is an independent determinant of creatinine clearance or urinary albumin excretion, stepwise multiple regression analysis was performed using other factors with a significant Pearson's correlation, together with blood viscosity as explanatory variables. Among them were age, body mass index (BMI), mean blood pressure (that is, diastolic blood pressure plus pulse pressure/3), pulse rate, triglyceride, HDL cholesterol, HOMA-IR and serum high-sensitive C-reactive protein. ${ }^{24-27}$ A $P$-value less than 0.05 was considered statistically significant.

\section{RESULTS}

Clinical characteristics of the patients

Clinical characteristics of the patients are summarized in Table 1. Creatinine clearance and urinary albumin excretion were $123 \pm 33$ $(60-208) \mathrm{ml} \mathrm{min}^{-1}$ per $1.73 \mathrm{~m}^{2}$ and $19 \pm 19$ (1-106) $\mathrm{mg}$ per day, respectively. Blood viscosity and plasma viscosity were $4.12 \pm 0.49$ $(3.06-5.80) \mathrm{mPa} \cdot \mathrm{S}$ and $1.74 \pm 0.08(1.53-1.95) \mathrm{mPa} \cdot \mathrm{S}$, respectively. There was no difference in blood viscosity and plasma viscosity between 23 EHT $(4.24 \pm 0.41 \mathrm{mPa} \cdot \mathrm{S}, 1.73 \pm 0.08 \mathrm{mPa} \cdot \mathrm{S})$ and the same number of age- and sex-matched normotensive subjects $(4.25 \pm 0.42 \mathrm{mPa} \cdot \mathrm{S}, 1.72 \pm 0.07 \mathrm{mPa} \cdot \mathrm{S})$.
Table 1 Clinical characteristics of the patients

\begin{tabular}{|c|c|}
\hline Age (years) & $56 \pm 12$ \\
\hline Sex (male/female) & $96 / 68$ \\
\hline Menopause & 48 \\
\hline Family history of hypertension & $96(59)$ \\
\hline Current smoker & $24(15)$ \\
\hline Height $(\mathrm{cm})$ & $162 \pm 10$ \\
\hline Body weight (kg) & $66 \pm 13$ \\
\hline Body mass index $\left(\mathrm{kg} \mathrm{m}^{-2}\right)$ & $24.8 \pm 4.0$ \\
\hline Blood pressure (mm Hg) & $155 \pm 19 / 93 \pm 14$ \\
\hline Pulse rate (beats per min) & $71 \pm 12$ \\
\hline Red blood cell count $\left(\times 10^{4} \mu \mathrm{l}^{-1}\right)$ & $439 \pm 41$ \\
\hline Hematocrit (\%) & $39.6 \pm 3.6$ \\
\hline Total protein $\left(\mathrm{gdl}^{-1}\right)$ & $6.6 \pm 0.4$ \\
\hline Total cholesterol (mg dl-1) & $193 \pm 42$ \\
\hline Triglyceride $\left(\mathrm{mg} \mathrm{dl}^{-1}\right)$ & $100 \pm 56$ \\
\hline HDL cholesterol (mg dl ${ }^{-1}$ ) & $53 \pm 13$ \\
\hline HOMA-IR & $1.64 \pm 1.82$ \\
\hline High-sensitive $\mathrm{C}$-reactive protein ( $\mathrm{mg} \mathrm{dl}^{-1}$ ) & $0.138 \pm 0.279$ \\
\hline Plasma nitrate and nitrite ( $\mu \mathrm{m})$ & $47.4 \pm 34.1$ \\
\hline Plasma fibrinogen (mg dl ${ }^{-1}$ ) & $240 \pm 58$ \\
\hline Serum creatinine $\left(\mathrm{mg} \mathrm{dl}^{-1}\right)$ & $0.68 \pm 0.16$ \\
\hline Urinary creatinine concentration ( $\mathrm{mg} \mathrm{dl}^{-1}$ ) & $92 \pm 53$ \\
\hline Urinary sodium concentration $\left(\mathrm{mEq} \mathrm{I} \mathrm{I}^{-1}\right.$ ) & $125 \pm 52$ \\
\hline
\end{tabular}

Values are mean \pm s.d. or number (\%) of patients.

Abbreviations: HDL, high-density lipoprotein; HOMA-IR, homeostasis model assessment index of insulin resistance.

\section{Associations of blood viscosity with anthropometric variables,} hematological variables and systemic hemodynamics

In all the patients, blood viscosity correlated positively with BMI $(r=0.261, P<0.001)$. In addition, blood viscosity correlated positively with hematocrit $(r=0.829, P<0.001)$ and plasma viscosity $(r=0.234$, $P=0.003)$. Plasma viscosity also correlated positively with triglyceride $(r=0.191, P=0.016)$, plasma fibrinogen $(r=0.402, P<0.001)$ and high-sensitive C-reactive protein $(r=0.424, P<0.001)$, but negatively with HDL cholesterol $(r=-0.226, P=0.004)$.

As for the association between hemorheological variables and systemic hermodynamics, blood viscosity correlated positively with mean blood pressure $(r=0.164, P=0.036)$ despite no relationship between blood viscosity and pulse rate.

\section{Associations of blood viscosity with renal function and urinary albumin excretion}

In all the patients, blood viscosity correlated negatively with creatinine clearance ( $r=-0.185, P=0.018$, Figure 1$)$ and positively with urinary albumin excretion $(r=0.253, P=0.001$, Figure 2$)$. These results indicated increased blood viscosity was associated with reduced renal function and increased albuminuria in EHT without chronic kidney disease. In addition, plasma viscosity correlated positively with urinary albumin excretion $(r=0.265, P<0.001$, Figure 2$)$, although there was no relationship between plasma viscosity and creatinine clearance $(r=0.100$, not significant, Figure 1$)$. Apart from blood viscosity, only serum triglyceride correlated negatively with creatinine clearance $(r=-0.187, P=0.017)$. BMI $(r=0.242, P=0.002)$, mean blood pressure $(r=0.268, P=0.001)$, pulse rate $(r=0.213, P=0.006)$, triglyceride $(r=0.229, P=0.003)$, HDL cholesterol $(r=-0.216$, $P=0.006)$, HOMA-IR $(r=0.183, \quad P=0.019)$ and high-sensitive C-reactive protein $(r=0.195, P=0.012)$ were associated with urinary albumin excretion. In contrast, there was no relationship between plasma $\mathrm{NO}_{\mathrm{x}}$ and urinary albumin excretion. Stepwise multiple regression analysis disclosed blood viscosity as an independent 

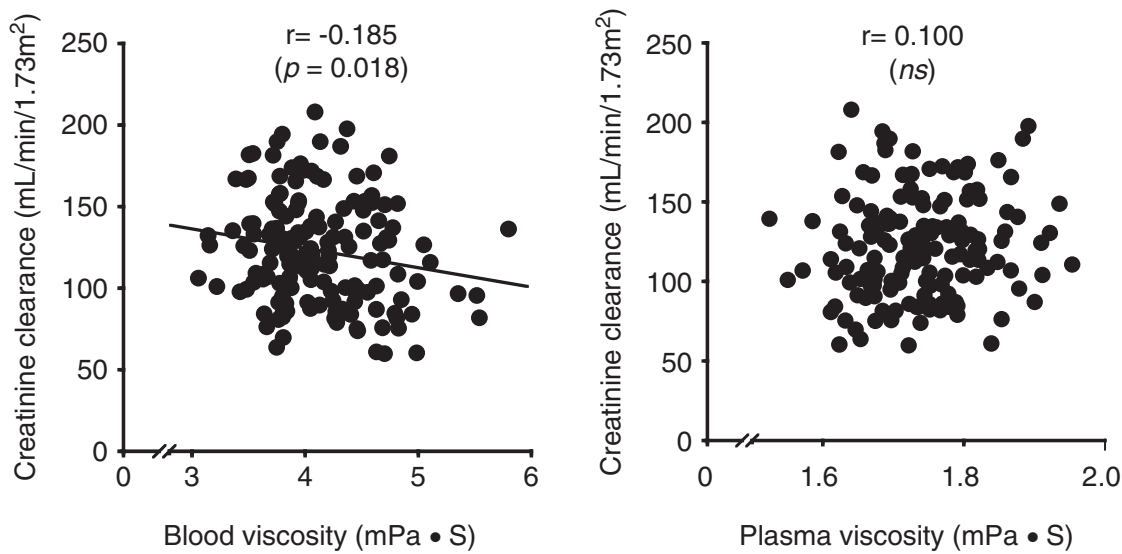

Figure 1 Correlations of blood viscosity (left panel) and plasma viscosity (right panel) with creatinine clearance. Blood viscosity, but not plasma viscosity, correlated negatively with creatinine clearance.
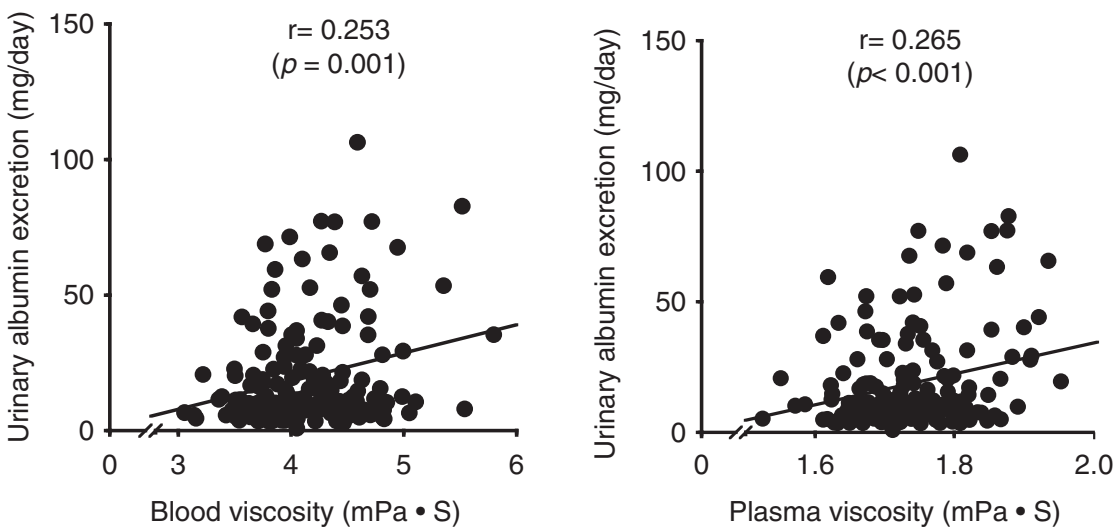

Figure 2 Correlations of blood viscosity (left panel) and plasma viscosity (right panel) with urinary albumin excretion. Both blood viscosity and plasma viscosity correlated positively with urinary albumin excretion.

Table 2 Stepwise multiple regression analysis of creatinine clearance or urinary albumin excretion.

\begin{tabular}{lcc}
\hline Variable & F-value & P-value \\
\hline Creatinine clearance $\left(\mathrm{R}^{2}=0.058\right)$ & & \\
$\quad$ Triglyceride & 3.98 & 0.048 \\
Blood viscosity & 3.89 & 0.050 \\
& & \\
Urinary albumin excretion $\left(\mathrm{R}^{2}=0.216\right)$ & & \\
Mean blood pressure & 4.44 & 0.037 \\
High-sensitive C-reactive protein & 4.02 & 0.047 \\
Blood viscosity & 4.28 & 0.040 \\
\hline
\end{tabular}

Stepwise multiple regression analysis of creatinine clearance was performed using triglyceride, together with blood viscosity as explanatory variables. Stepwise multiple regression analysis of urinary albumin excretion was performed using body mass index, mean blood pressure, pulse rate, triglyceride, HDL cholesterol, HOMA-IR and high-sensitive C-reactive protein together with blood viscosity as explanatory variables.

Abbreviations: HDL, high-density lipoprotein; HOMA-IR, homeostasis model assessment index of insulin resistance.

determinant of creatinine clearance (multiple $R^{2}=0.058$ ) and urinary albumin excretion (multiple $R^{2}=0.216$, Table 2).

\section{DISCUSSION}

The present study was the first to determine the associations of blood viscosity with renal function and urinary albumin excretion before the onset of renal damage in EHT. The major findings of the study are as follows. Increased blood viscosity correlated with reduced renal function and elevated urinary albumin excretion. Furthermore, blood viscosity was an independent determinant of renal function and albuminuria in EHT.

Factors possibly determining blood viscosity in EHT

Increased blood viscosity is often found in patients with obesity or metabolic syndrome. ${ }^{16,21}$ Blood viscosity also depends on plasma viscosity, red blood cell rigidity and hematocrit. ${ }^{21,23}$ By contrast, plasma viscosity is affected by dyslipidemia, insulin resistance, inflammation and plasma fibrinogen. ${ }^{16-19}$ In the present study, blood viscosity correlated positively with BMI, plasma viscosity and hematocrit, although red blood cell rigidity was not evaluated. Moreover, plasma viscosity correlated positively with triglyceride, plasma fibrinogen and high-sensitive C-reactive protein, but negatively with HDL cholesterol, findings consistent with previous reports. ${ }^{16-19}$ From these results, blood viscosity could be determined by extent of obesity, hemoconcentration and plasma viscosity in EHT without apparent renal disease.

Effects of increased blood viscosity on the kidney in EHT It is beyond the scope of this study to establish the causal relationship between increased blood viscosity and reduced renal function or increased albuminuria in EHT without apparent renal damage. However, our results could be explained as follows. According to the Hagen-Poiseuille law, increased blood viscosity results in reduced 
blood flow and increased vascular resistance in the renal vascular beds. ${ }^{1}$ In particular, blood viscosity-induced elevation of vascular resistance may be most prominent in the efferent arterioles of the kidney because of hemoconcentration due to glomerular filtration. ${ }^{4,5}$ Elevated vascular resistance in the efferent arterioles could increase back pressure toward the glomerular vessels, thereby elevating intraglomerular pressure to maintain glomerular filtration in the face of reduced renal blood flow. ${ }^{4,5}$

Glomerular filtration rate may be affected by the above-mentioned alterations in renal blood flow and intraglomerular pressure. ${ }^{28}$ Namely, blood viscosity-mediated reduction in renal blood flow increases the transglomerular difference of oncotic pressure during filtration, thereby decreasing glomerular ultrafiltration pressure and glomerular filtration rate. ${ }^{29}$ By contrast, blood viscosity-mediated elevation in intraglomerular pressure increases the transglomerular hydraulic pressure gradient during filtration, thereby increasing glomerular ultrafiltration pressure and glomerular filtration rate. In the present study, the net effect of increases in blood viscosity could lead to a reduction in creatinine clearance. Additionally, blood viscosity-mediated elevation in efferent arteriolar resistance as well as systemic blood pressure may increase intraglomerular pressure and thereby alter glomerular permeability against protein, resulting in increased urinary albumin excretion. ${ }^{4,5,26,27}$ Elevated vascular resistance in the efferent arterioles may also reduce the peritubular capillary blood flow, thereby causing interstitial ischemia, ${ }^{4-6}$ as reported in contrast-induced nephropathy. ${ }^{9,10}$ Indeed, these consecutive events could explain the possible mechanisms by which increased blood viscosity can affect the renal vascular beds in EHT without renal damage. However, it is well known that in EHT, intraglomerular pressure is not always elevated owing to an enhanced vasoconstriction at the preglomerular resistance vessels. ${ }^{30}$ Furthermore, glomerular ischemia induced by preglomerular vasoconstriction is unlikely to increase urinary albumin excretion in EHT. Therefore, renal hemodynamics has to be examined to draw a definitive conclusion of the mechanism of blood viscosity-induced influences on renal vasculature in EHT.

In the present study, blood viscosity was associated with creatinine clearance but plasma viscosity was not. In the microcirculation such as glomerular capillaries, the corpuscular elements of blood tend to migrate to the centerline of flow and plasma layer is in direct contact with vascular wall. ${ }^{31}$ Therefore, it seems likely that plasma viscosity rather than blood viscosity could affect glomerular capillary flow and glomerular filtration. However, this was not the case in the present result.

\section{Clinical implications}

Reduced renal function and/or elevated albuminuria reflect widespread cardiovascular damages as well as glomerular impairment, indicating an increased risk of future cardiovascular and renal disease. $^{32-35}$ In the present study, increased blood viscosity was associated with reduced renal function and increased urinary albumin excretion in EHT without renal disease, although the hemorheological variables were not different between EHT and normotensive subjects. This finding indicates that increased blood viscosity might be harmful sufficiently enough to generate renal damage in EHT in contrast to normotensive healthy subjects.

Along with the development of renal injuries, renal anemia could occur and accelerate renal ischemia via reduction in oxygen supply in EHT. Therefore, correction of renal anemia could be recommended to relieve the decreased delivery of oxygen and protect kidneys from ischemic injuries. ${ }^{36}$ However, clinical studies demonstrated that correction of anemia using erythropoietin therapy was not proven to have cardiovascular protective effects in patients with chronic kidney disease. ${ }^{36}$ In addition, incidence of cardiovascular events was greater especially in the patients treated with the higher target of hematocrit. ${ }^{37}$ This finding supports that hyperviscosity induced by erythropoietin therapy could reduce blood flow, resulting in ischemic damages in vital organs. Thus, increased blood viscosity is considered as one of the important cardiovascular risk factors in the advanced stage as well as early stage of essential hypertension.

\section{Study limitations}

The present study was limited for several reasons. First, we were unable to confirm a causal relationship between increased blood viscosity and renal dysfunction or albuminuria in EHT because of the observational nature of this study. However, our results do suggest a negative influence of blood viscosity on the renal vascular beds before the onset of apparent renal disease in EHT. Further longitudinal studies will be required to explore the clinical implications of blood viscosity on the pathogenesis of renal injuries in EHT. Second, therapeutic interventions affecting blood hemorheology should be investigated to determine whether they are effective in preventing renal damage. Especially, alpha-1 blockers could be recommended because they reduce not only blood viscosity but also albuminuria in EHT. ${ }^{38,39}$ Third, our data did not support the notion that increased urinary albumin excretion could reflect systemic endothelial dysfunction in EHT. $^{40}$ Because the endothelial function was estimated only by plasma $\mathrm{NO}_{\mathrm{x}}$ in our study, the measurements of other substances released from endothelial cells, such as prostacyclin, or blood flow during reactive hyperemia should have been performed for the precise evaluation of endothelial functions. ${ }^{41,42}$ Fourth, in the present study, the value of creatinine clearance seemed to be relatively high according to their age, possibly due to increase in the tubular secretion of creatinine into urine. ${ }^{43}$ The accurate and reliable method such as inulin clearance should have been employed for the measurement of glomerular filtration rate. Finally, EHT of advanced stages with kidney disease should be studied, because the patients with kidney disease could be associated with increased blood viscosity. ${ }^{44}$

Although limited for these reasons, the results of this study indicate that increased blood viscosity is a risk for renal damage before the development of kidney disease in EHT.

\section{CONFLICT OF INTEREST}

The authors declare no conflict of interest.

1 Lowe GD. Blood rheology in arterial disease. Clin Sci 1986; 71: 137-146.

2 Kwaan HC, Bongu A. The hyperviscosity syndromes. Semin Thromb Hemost 1999; 25 199-208.

3 Velcheva I, Antonova N, Titianova E, Damianov P, Dimitrov N, Dimitrova V. Hemorheological disturbances in cerebrovascular diseases. Clin Hemorheol Microcirc 2008; 39: 391-396.

4 Simpson LO. A hypothesis proposing increased blood viscosity as a cause of proteinuria and increased vascular permeability. Nephron 1982; 31: 89-93.

5 Simpson LO. Increased blood viscosity as the pathogenic agent in minimal change nephrosis: a new hypothesis. Med Hypotheses 1981; 7: 1421-1429.

6 Dittrich S, Kurschat K, Lange PE. Abnormal rheology in cyanotic congenital heart disease-a factor in non-immune nephropathy. Scand J Urol Nephrol 2001; 35: 411-415.

7 Simpson LO, Shand BI, Olds RJ. A reappraisal of the influence of blood rheology on glomerular filtration and its role in the pathogenesis of diabetic nephropathy. J Diabet Complications 1987; 1: 137-144.

8 Solerte SB, Fioravanti M, Pezza N, Locatelli M, Schifino N, Cerutti N, Severgnini S, Rondanelli M, Ferrari E. Hyperviscosity and microproteinuria in central obesity: relevance to cardiovascular risk. Int J Obes Relat Metab Disord 1997; 21: 417-423. 
9 Seeliger E, Flemming B, Wronski T, Ladwig M, Arakelyan K, Godes M, Möckel M, Persson PB. Viscosity of contrast media perturbs renal hemodynamics. J Am Soc Nephrol 2007; 18: 2912-2920.

10 Persson PB, Hansell P, Liss P. Pathophysiology of contrast medium-induced nephropathy. Kidney Int 2005; 68: 14-22.

11 Bogar L. Hemorheology and hypertension: not "chicken or egg" but two chickens from similar eggs. Clin Hemorheol Microcirc 2002; 26: 81-83.

12 Chabanel A, Chien S. Blood viscosity in human hypertension. In: Laragh JH and Brenner BM (eds) Hypertension: Pathophysiology, Diagnosis, and Management. 2nd edn, pp 365-376. (Raven Press, New York, 1995).

13 Smith WC, Lowe GD, Lee AJ, Tunstall-Pedoe H. Rheological determinants of blood pressure in a Scottish adult population. J Hypertens 1992; 10: 467-472.

14 Devereux RB, Drayer JI, Chien S, Pickering TG, Letcher RL, DeYoung JL, Sealey JE, Laragh $\mathrm{JH}$. Whole blood viscosity as a determinant of cardiac hypertrophy in systemic hypertension. Am J Cardiol 1984; 54: 592-595.

15 National Kidney Foundation. K/DOQI clinical practice guidelines for chronic kidney disease: evaluation, classification, and stratification. Am J Kidney Dis, 2002; 39(2 Suppl 1): S1-S266.

16 Zhang L, Pu K, Zhang SY, Ren WQ. Blood rheological properties are strongly related to the metabolic syndrome in middle-aged Chinese. Int J Cardiol 2006; 112: 229-233.

17 Brun JF. Hormones, metabolism and body composition as major determinants of blood rheology: potential pathophysiological meaning. Clin Hemorheol Microcirc 2002; 26: 63-79.

18 Contreras T, Vaya A, Palanca S, Sola E, Corella D, Aznar J. Influence of plasmatic lipids on the hemorheological profile in healthy adults. Clin Hemorheol Microcirc 2004; 30: 423-425.

19 Wannamethee SG, Lowe GD, Shaper AG, Rumley A, Lennon L, Whincup PH. The metabolic syndrome and insulin resistance: relationship to haemostatic and inflammatory markers in older non-diabetic men. Atherosclerosis 2005; 181: 101-108.

20 Staessen J, Broughton PM, Fletcher AE, Markowe HL, Marmot MG, Rose G, Semmence A, Shipley MJ, Bulpitt CJ. The assessment of the relationship between blood pressure and sodium intake using whole-day, daytime and overnight urine collections. J Hypertens 1991; 9: 1035-1040.

21 Sugimori $\mathrm{H}$, Tomoda F, Koike T, Kinuno H, Kurosaki H, Masutani T, Inoue H. Blood rheology and platelet function in untreated early-stage essential hypertensives complicated with metabolic syndrome. Int J Hypertens 2012; 2012: 109830.

22 Doffin J, Perrault R, Garnaud G. Blood viscosity measurements in both extensional and shear flow by a falling ball viscometer. Biorheology Supp/ 1984; 1: 89-93.

23 Fons C, Brun JF, Supparo I, Mallard C, Bardet L, Obsetti A. Evaluation of blood viscosity at high shear rate with a falling ball viscometer. Clin Hemorheol 1993; 13: 651-659.

24 Ruan X, Guan Y. Metabolic syndrome and chronic kidney disease. J Diabetes 2009; 1: 236-245.

25 Menne J, Chatzikyrkou C, Haller H. Microalbuminuria as a risk factor: the influence of renin-angiotensin system blockade. J Hypertens 2010; 28: 1983-1994.

26 Palatini P. Microalbuminuria in hypertension. Curr Hypertens Rep 2003; 5: 208-214.

27 Comper WD, Hilliard LM, Nikolic-Paterson DJ, Russo LM. Disease-dependent mechanisms of albuminuria. Am J Physiol Renal Physiol 2008; 295: F1589-F1600.

28 Baylis C. Glomerular filtration dynamics. In Lote CJ (ed) Advances in Renal Physiology. pp 33-83. (Croom Helm, Austria, 1986)
29 Brenner BM, Troy JL, Daugharty TM, Deen WM, Robertson CR. Dynamics of glomerular ultrafiltration in the rat. II. Plasma-flow dependence of GFR. Am J Physiol 1972; 223: 1184-1190.

30 Luke RG. Hypertensive nephrosclerosis: pathogenesis and prevalence. Essential hypertension is an important cause of end-stage renal disease. Nephrol Dial Transplant 1999; 14: 2271-2278.

31 McHedlishvili G, Maeda N. Blood flow structure related to red cell flow: determinant of blood fluidity in narrow microvessels. Jpn J Physiol 2001; 51: 19-30.

32 Cerasola G, Cottone S. Mulè. The progressive pathway of microalbuminuria: from early marker of renal damage to strong cardiovascular risk predictor. J Hypertens 2010; 28: 2357-2369.

33 Ruggenenti P, Remuzzi G. Time to abandon microalbuminuria? Kidney Int 2006; 70 : 1214-1222.

34 Go AS, Chertow GM, Fan D, McCulloch CE, Hsu CY. Chronic kidney disease and the risks of death, cardiovascular events, and hospitalization. N Engl J Med 2004; 351: 1296-1305.

35 Keith DS, Nichols GA, Gullion CM, Brown JB, Smith DH. Longitudinal follow-up and outcomes among a population with chronic kidney disease in a large managed care organization. Arch Intern Med 2004; 164: 659-663.

36 Jeong SK, Cho YI, Duey M, Rosenson RS. Cardiovascular risks of anemia correction with erythrocyte stimulating agents: should blood viscosity be monitored for risk assessment? Cardiovasc Drugs Ther 2010; 24: 151-160.

37 Besarab A, Bolton WK, Browne JK, Egrie JC, Nissenson AR, Okamoto DM, Schwab SJ, Goodkin DA. The effects of normal as compared with low hematocrit values in patients with cardiac disease who are receiving hemodialysis and epoetin. N Eng/ J Med 1998; 339: 584-590.

38 Toth K, Kesmarky G, Vekasi J, Nemes J, Czopf L, Kapronczay P, Halmosi R, Papp E, Juricskay I. Hemorheological and hemodynamic parameters in patients with essential hypertension and their modification by alpha- 1 inhibitor drug treatment. Clin Hemorheol Microcirc 1999; 21: 209-216.

39 Kario K, Matsui Y, Shibasaki S, Eguchi K, Ishikawa J, Hoshide S, Ishikawa S, Kabutoya T, Schwartz JE, Pickering TG, Shimada K. Japan Morning Surge-1 (JMS-1) Study Group. An alpha-adrenergic blocker titrated by self-measured blood pressure recordings lowered blood pressure and microalbuminuria in patients with morning hypertension: the Japan Morning Surge-1 Study. J Hypertens 2008; 26: 1257-1265.

40 Pedrinelli R, Giampietro O, Carmassi F, Melillo E, Dell'Omo G, Catapano G, Matteucci E, Talarico L, Morale M, De Negri F, De Bello V. Microalbuminuria and endothelial dysfunction in essential hypertension. Lancet 1994; 344: 14-18.

41 Frangos JA, Eskin SG, McIntire LV, Ives CL. Flow effects on prostacyclin production by cultured human endothelial cells. Science 1985; 227: 1477-1479.

42 Costa F, Sulur P, Angel M, Cavalcante J, Haile V, Christman B, Biaggioni I. Intravascular source of adenosine during forearm ischemia in humans: implications for reactive hyperemia. Hypertension 1999; 33: 1453-1457.

43 Prié D. Friedlander G. The clinical assessment of renal function. In Davison $A M$ Cameron JT, Grünfeld JP, Ponticelli C, Ritz E, Winearls CG and van Ypersele C (eds) Clinical Nephrology. 3rd edn, pp 47-72. (Oxford University Press, New York, 2004).

44 Brimble KS, McFarlane A, Winegard N, Crowther M, Churchill DN. Effect of chronic kidney disease on red blood cell rheology. Clin Hemorheol Microcirc 2006; 34: $411-420$. 